\title{
Determinantes de divulgación de desempeño ambiental y economía circular de empresas peruanas
}

\section{Determinants of environmental performance and circular economy disclosure of Peruvian companies}

\author{
Julio Hernández Pajares ${ }^{1}$, Valeria Yagui Nishii ${ }^{2}$
}

\section{RESUMEN}

Es una realidad el creciente desarrollo del desempeño e información de desempeño ambiental en empresas peruanas, debido a la influencia institucional de la normativa internacional principalmente en organizaciones de actividades con mayor impacto de sostenibilidad, sin embargo, las prácticas de economía circular (EC) como concepto de desarrollo sostenible no presentan el mismo impulso.

Esta investigación busca analizar el nivel de cumplimiento de los indicadores de desempeño ambiental de acuerdo con la GRI y las prácticas de EC, y su relación con el tamaño y sector para empresas peruanas que han publicado su reporte de sostenibilidad voluntario para el año 2016.

Esta investigación cuantitativa tiene un alcance descriptivo y relacional, para ello se realiza un análisis de contenido de los reportes de sostenibilidad de las empresas para examinar las variables señaladas.

Los resultados señalan un mayor cumplimiento de indicadores de gestión ambiental de recursos, transporte y cumplimiento regulatorio, por otro lado, de las prácticas de EC la reducción de emisiones e insumos son las más divulgadas. El sector empresarial y el tamaño de las empresas solo encuentran relación con el nivel de información ambiental. La divulgación de prácticas de EC es menor que las prácticas ambientales y los aspectos revelados no presentan la implementación de sistemas de EC en su mayoría.

Palabras clave: economía circular, Global Reporting Initiative, GRI, reporte de sostenibilidad, Perú.

Recepción: 29/10/2020. Aprobación: 27/12/2020.

1 Universidad de Piura, Facultad de Ciencias Económicas y Empresariales, Lima, Perú, julio.hernandez@udep.edu.pe

2 Universidad de Piura, Facultad de Ciencias Económicas y Empresariales, Lima, Perú, valeria.yagui.n@gmail.com 


\begin{abstract}
The growing development of performance and environmental performance disclosure in Peruvian companies is a reality. This is due to the institutional influence of the international normative seen mainly in organizations with activities of a greater sustainability impact. However, circular economy (CE) practices, as a concept of sustainable development, do not present the same momentum.

This research seeks to analyse the level of compliance with the environmental performance indicators, in accordance with the GRI and EC practices, and its relationship with the size and sector of Peruvian companies that have published their voluntary sustainability report for 2016 .

This quantitative research has a descriptive and relational scope, for which a content analysis of the sustainability reports of the companies was carried out to examine the variables indicated.

The results indicate a greater compliance with indicators of the environmental management of resources, transportation and regulatory compliance. Meanwhile, of the EC practices the reduction of emissions and inputs are the most publicized. The business sector and the size of the company is only related to the level of environmental information. The disclosure of CE practices is less than environmental practices and the aspects disclosed do not, in the main, present the implementation of CE systems.
\end{abstract}

Keywords: circular economy, Global Reporting Initiative, GRI, sustainability report, Peru.

\title{
INTRODUCCIÓN
}

La información de sostenibilidad es un elemento importante para las organizaciones dado que les permiten divulgar a sus partes interesadas de su triple desempeño económico, ambiental y social como parte de sus objetivos estratégicos. Asimismo, pueden obtener una ventaja competitiva aumentando la eficiencia de los procesos y productos y mejorando la confianza con sus stakeholders, logrando la lealtad de clientes, proveedores, colaboradores y proporcionando a los inversionistas información para sus decisiones (Landrum, 2017; Landrum \& Ohsowski, 2018; Moneva et al., 2019).

La práctica más difundida de información de sostenibilidad ha sido la que propone la Global Reporting Initiative (GRI). Investigaciones internacionales encuentran una mayor divulgación de reportes de sostenibilidad bajo este modelo en empresas cuyas actividades presentan mayor impacto de sostenibilidad y con mayor presencia en mercados de valores (Kolk et al., 2001; Marimon et al., 2012; Alonso-Almeida, 2015; Landrum \& Ohsowski, 2018).

En América Latina los estudios encuentran que el nivel de información de sostenibilidad es destacado para las empresas con mayor actividad ambiental y social en sectores de minería, energía, manufactura y agroindustria con una significativa influencia institucional de organizaciones locales y transnacionales (Aranguren \& Maldonado, 2019; Calixto, 2013; Del Barco et al., 2018; Rabasedas, 2018; Hernández-Pajares, 2018).

Por otro lado, importantes investigaciones consideran la teoría institucional y de legitimidad para explicar la naturaleza de divulgación de información ambiental de las empresas. De acuerdo 
a la teoría institucional las motivaciones del reporte de sostenibilidad, en entornos normativos donde las empresas por un isomorfismo mimético informan de forma similar que sus empresas pares para competir en la obtención de legitimidad, obedece más a una influencia de regulación local y extranjera (De Villiers \& Alexander, 2014).

Según la teoría de legitimidad el reporte de sostenibilidad busca informar positivamente y cumplir dicho contrato cumpliendo los valores o normas sociales que pueden influir en la percepción de los grupos de interés como mantener sus relaciones con los clientes, sociedad y mejorar su legitimidad corporativa (Deegan, 2002; Husillos, 2007; O’Donovan, 2002).

Pero el desarrollo sostenible también se ve afectado por los problemas de uso recursos y su impacto ambiental, por lo que es una elección una economía circular (EC) sin contaminación y emisiones y reutilización de recursos, que ha presentado un interés de las empresas los últimos años en países occidentales y China principalmente, por influencia regulatoria de los gobiernos o una búsqueda de una innovación productividad y mejora de procesos, pero que aún se encuentran en desarrollo y no llega a integrarse a los sistemas y estrategias de desarrollo sostenible (Linder, M., \& Williander, 2017; Murray et al., 2015; Wang et al., 2014). También es una realidad la falta de indicadores y la medición del desempeño de sostenibilidad con enfoques circulares, por otro lado, aunque no existe un estándar, se han presentado esfuerzos de divulgación de prácticas de EC, inclusive bajo el modelo de reporte integrado, como transparencia a sus grupos de interés y en la creación de valor financiero (Barnabè y Nazir, 2020; Kuo \& Chang, 2021; Stewart \& Niero, 2018).

Las prácticas de (EC), son un tema relevante para las organizaciones y gobiernos, por representar una alternativa viable al modelo lineal de producción que permite lograr un compromiso entre productividad y utilización de recursos de circularmente en los procesos (Bocken et al., 2016; Moneva et al., 2018; Stewart y Niero, 2018; Yuan et al., 2006). Aunque a nivel mundial esta práctica se encuentra muy difundida e investigada, en el Perú se encuentra en desarrollo por las organizaciones y demanda de un incentivo institucional (Geng et al., 2012; Ghisellini et al., 2016). En ese sentido, el gobierno peruano ha promulgado una regulación denominada "Hoja de ruta hacia una Economía Circular en el sector industria" para incentivar la sostenibilidad ambiental en las actividades económicas promoviendo la EC en los mercados y la generación de soluciones sostenibles para el desarrollo en la producción de las empresas (PRODUCE, 2020). La viabilidad de la economía circular en la región de Latinoamérica es un reto, considerando que presenta una economía extractiva y no industrializada, por lo que su implementación requiere analizar las realidades socioculturales, político-económicas de la región (Paño-Yáñez, 2021). Lo anterior lleva al motivo de esta investigación en un aporte sobre el conocimiento y reporte de las prácticas de EC en empresas de un país en vías de desarrollo, donde la influencia institucional no alcanza los niveles de los países desarrollados.

\section{Marco Teórico}

\section{Información de sostenibilidad y ambiental}

Investigaciones consideran la teoría de legitimidad e institucional para explicar el reporte ambiental y de sostenibilidad. De acuerdo al enfoque de legitimidad, la divulgación ambiental es una forma de legitimar la existencia continua y operaciones de las empresas, respondiendo a sus 
obligaciones con la sociedad, mediante una imagen de un buen desempeño ambiental, en actividades con mayor impacto de sostenibilidad (Cormier et al., 2005; Deegan et al., 2002; Husillos, 2007; Neu et al., 1998). Esta búsqueda de legitimidad tiene como objetivo incidir en las percepciones y expectativas sociales, respaldar la reputación ambiental o desviar la atención de impactos negativos en el medio ambiente (Deegan, 2002; O’Donovan, 2002).

Desde el enfoque institucional las empresas desarrollan una divulgación similar a otras empresas o de acuerdo a lo que la normativa exige por influencia de asociaciones empresariales y profesionales, entes reguladores locales $u$ organizaciones no gubernamentales internacionales que promueven sus estándares una divulgación armonizada, como el Pacto Mundial, GRI Standards e ISO 26000 (De Villiers \& Alexander, 2014; Neu et al., 1998).

Por otro lado, respecto a los factores de divulgación, las empresas de mayor tamaño están incentivadas a una importante divulgación de información de sostenibilidad ya que están más expuestas socialmente y buscan legitimarse, asimismo tiene más presiones por parte de la sociedad y de los grupos de interés de rendir cuenta sobre su desempeño de sostenibilidad, además de disponer de más recursos y menores costos para elaborar dicho reporte (Da Silva \& Aibar, 2011; D'Amico et al., 2016), lo anterior se confirma en investigaciones que encuentran que el tamaño de las organizaciones resulta un factor determinante en la información de desempeño ambiental (Bhattacharyya, 2014; Kuzey \& Uyar, 2017; Kouloukoui et al., 2018; Orazalin \& Mahmood, 2019).

Respecto al sector empresarial, el tipo de actividades económicas resulta ser determinante en el nivel de información ambiental, debido al nivel de impacto en el medio ambiente como empresas de energía, mineras e industriales, que presentan una significativa divulgación para legitimar sus actividades frente a la sociedad (Cormier \& Magnan, 2003; Kouloukoui et al., 2018; Orazalin \& Mahmood, 2019). Investigaciones para empresas a nivel internacional y de Latinoamérica el sector resulta un factor determinante en el nivel de información ambiental (Bhattacharyya, 2014; Baalouch et al., 2019; Bednárová et al., 2019; Dyduch \& Krasodomska, 2017; Hernández-Pajares et al., 2017).

\section{Prácticas de Economía Circular}

Las investigaciones sobre EC se han centrado en la propuesta de modelos de gestión y en sus prácticas, sin embargo, su estudio como modelo de estrategia y gestión en las empresas aún se encuentra en desarrollo (Aranda-Usón et al., 2020). Los objetivos de la EC son la reducción del flujo de materiales, la eficiencia, la renovación constante del capital natural y gestionar ciclos de materiales de alto valor en lugar del reciclaje tradicional (Ghisellini et al., 2016; Yuan et al., 2006). La EC se presenta en sus investigaciones como una combinación de actividades con distintitas perspectivas y no como un enfoque sistémico; no todas las definiciones muestran vínculos explícitos con la de sostenibilidad, aunque la EC se relaciona con la gestión de calidad ambiental; producción eco-innovadora sostenible, su relación con el desarrollo sostenible no es clara, además de las limitaciones que se presentan en las empresas por la falta de conocimiento, financiación para inversiones y gestión de reciclado (Kirchherr et al., 2017; Prieto-Sandoval et al., 2018; Scarpellini et al., 2019).

Sin embargo, otros estudios encuentran relación entre el desempeño de sostenibilidad y la EC, 
dado que ambos conceptos integran los aspectos no financieros en el desempeño, fomentan un cambio en la gestión de recursos y capacidades y promueven la innovación en los procesos (Sehnem et al., 2019; Geissdoerfer et al., 2017). Asimismo, el modelo de indicadores ambientales de la GRI se ha considerado para la medición de las prácticas de EC como señalan las investigaciones de Li et al. (2019), Veleva et al. (2017) y Janik et al. (2020).

Las investigaciones sobre estrategias y prácticas de EC en el Perú, la consideran como una alternativa de contribución de las empresas al desarrollo sostenible mediante una mejora en la gestión de la calidad y eco eficiencia (Alvarez-Risco, 2020; Laso et al., 2018), por otro lado, la investigación de Alverez-Risco et al. (2021) encuentra que la divulgación en reportes de sostenibilidad corresponden a declaraciones sobre objetivos estratégicos en prácticas de EC, pero con una menor información de gestión.

De acuerdo a la literatura, diversos aspectos se han considerado como prácticas de EC en las organizaciones, la Tabla 1 muestra dichos aspectos que se consideran en los estudios.

\section{Tabla 1}

\section{Prácticas de EC según la revisión literaria.}

\begin{tabular}{|c|c|}
\hline Práctica de EC & Autores \\
\hline $\begin{array}{l}\text { Reducción de insumos y } \\
\text { menor utilización de recursos } \\
\text { naturales }\end{array}$ & $\begin{array}{l}\text { Barnabè \& Nazir (2020); Cerdá \& Khalilova (2016); Luttropp \& } \\
\text { Lagerstedt (2006); Elia et al. (2017); Geng et al. (2012); Kirchherr } \\
\text { et al. (2017); Murray et al (2015); Potting et al. (2017); Bocken et al. } \\
\text { (2016) }\end{array}$ \\
\hline $\begin{array}{l}\text { Reducción de } \\
\text { emisiones }\end{array}$ & $\begin{array}{l}\text { Barnabè \& Nazir (2020); Cerdá \& Khalilova (2016); Elia et al. (2017); } \\
\text { Geng et al. (2012); Stewart \& Niero (2018), Wang et al. (2014). }\end{array}$ \\
\hline $\begin{array}{l}\text { Disminución de generación } \\
\text { materiales y residuos }\end{array}$ & Cerdá y Khalilova (2016); Geng et al. (2012); Kirchherr et al. (2017) \\
\hline $\begin{array}{l}\text { Reemplazar los recursos no } \\
\text { renovables por renovables }\end{array}$ & $\begin{array}{l}\text { Cerdá \& Khalilova (2016); Moneva et al. (2018); Ghisellini et al. } \\
\text { (2016); Prieto-Sandoval et al. (2018); Bocken et al. (2016); Elia et al. } \\
\text { (2017) }\end{array}$ \\
\hline $\begin{array}{l}\text { Mayor proporción de materiales } \\
\text { reciclables y reciclados } \\
\text { que puedan reemplazar a } \\
\text { materiales vírgenes }\end{array}$ & Cerdá y Khalilova (2016); Elia et al. (2017); Stewart \& Niero (2018). \\
\hline $\begin{array}{l}\text { Reutilización / reciclaje de los } \\
\text { residuos generados }\end{array}$ & $\begin{array}{l}\text { Cerdá \& Khalilova (2016); Lacy \& Rutqvist (2015); Moneva et al. } \\
\text { (2018); Murray et al (2015); Elia et al. (2017); Geng et al. (2012); } \\
\text { Ghisellini et al. (2016); Kirchherr et al. (2017); Potting et al. (2017); } \\
\text { Prieto-Sandoval et al. (2018); Bocken et al. (2016); Aranda-Usón } \\
\text { (2020); Stewart \& Niero (2018). }\end{array}$ \\
\hline Eco Diseño de Procesos & $\begin{array}{l}\text { Barnabè \& Nazir (2020) Cerdá \& Khalilova (2016); Luttropp \& } \\
\text { Lagerstedt (2006); Lacy \& Rutqvist (2015); Moneva et al. (2018), Elia } \\
\text { et al. (2017); Ghisellini et al. (2016); Kirchherr et al. (2017); Potting et } \\
\text { al. (2017); Bocken et al. (2016); Aranda-Usón (2020). }\end{array}$ \\
\hline
\end{tabular}

Fuente: Elaboración propia basada en revisión literaria. 
La revisión teórica lleva a plantear como problema de investigación, cuál es la naturaleza y nivel de cumplimiento de los indicadores de desempeño ambiental de acuerdo al GRI y, por otro lado, conocer el grado de divulgación de las prácticas de economía circular, señaladas en la teoría, considerando como determinantes el tamaño y tipo de actividad empresarial, de las empresas peruanas que han publicado sus reportes de sostenibilidad en la base de datos del GRI. Asimismo, se cuestiona si existe una influencia institucional de organizaciones y entes reguladores en el nivel de divulgación de desempeño ambiental y de EC.

Por lo anterior, se plantean como objetivos de esta investigación los siguientes:

a) Conocer y describir la naturaleza de las prácticas de desempeño ambiental de acuerdo a los indicadores del GRI y de EC según la revisión teórica para empresas peruanas que han publicado sus reportes según la guía GRI en el año 2016.

b) Describir el nivel de cumplimiento de los indicadores ambientales de acuerdo a la Guía G4 del GRI, y el nivel de divulgación de prácticas de EC para empresas peruanas que han publicado sus reportes según la guía GRI en el año 2016.

c) Analizar la influencia del tamaño de las empresas y su tipo de actividad en los niveles de información de desempeño ambiental y de EC.

d) Analizar la significatividad de la relación entre tamaño de las empresas y su tipo de actividad con los niveles de información de desempeño ambiental y de EC.

Para cumplir el objetivo de análisis de influencia y relación de las variables de tamaño y tipo de actividad con las variables de nivel de información de desempeño ambiental y de EC, se proponen las siguientes hipótesis:

H1: El sector empresarial incide positiva y significativamente en el nivel de cumplimiento de información ambiental.

H2: El sector empresarial incide positiva y significativamente en el nivel de información de prácticas de EC.

H3: El sector empresarial y tamaño se correlacionan positivamente con el nivel de cumplimiento de información ambiental.

H4: El sector empresarial y tamaño se correlacionan positivamente con el nivel de información de prácticas de EC.

\section{METODOLOGÍA, MATERIALES Y MÉTODOS}

La investigación se realizó mediante un estudio cuantitativo de naturaleza exploratoria y con alcance descriptivo de las variables de nivel de cumplimiento de los aspectos de desempeño ambiental y de prácticas de EC. Además, consideró un alcance relacional para verificar si existió incidencia y correlación entre las variables independientes de tamaño y sector empresarial con las variables dependientes de información de desempeño ambiental y de EC. 
La investigación se realizó siguiendo los siguientes pasos, primero, se determinó la muestra de empresas que publicaron su reporte de sostenibilidad para el periodo 2016 de la base de datos del GRI. Para dicha muestra se realizó una selección de los reportes de sostenibilidad publicados por dichas empresas en el periodo señalado.

Posteriormente se realizó un análisis de contenido para obtener las variables independientes de tamaño, de acuerdo al número de trabajadores y tipo de actividad, según la clasificación del GRI; asimismo, se realizó una medición de nivel de información de las variables dependientes de desempeño ambiental, según indicadores del GRI y de prácticas de EC.

Finalmente se realizó el análisis descriptivo de las variables, de incidencia significativa del tamaño y tipo de actividad en los niveles de información de desempeño ambiental y de EC mediante la prueba de Kruskal Wallis, y relacional mediante una prueba de correlación de Spearman entre las variables independientes y dependientes señaladas.

\section{Muestra}

Para el estudio se realizó una selección de las empresas peruanas que publicaron sus memorias de sostenibilidad en la base de datos de la GRI publicados el año 2016 por desempeños del 2015 y / o 2016, último periodo disponible, a la fecha de la investigación, de información completa según el Sustainability Disclosure Database (GRI, 2019). La muestra final consistió en 67 empresas. Esta muestra de empresas peruanas que publicaron reportes de sostenibilidad voluntario comprendió las empresas descritas en la Tabla 2. Se revisaron 58 reportes bajo la Guía G4 de la GRI y 9 reportes de sostenibilidad presentadas bajo otro modelo no GRI, obtenido de la base de datos o de la página web corporativa.

Tabla 2

Muestra clasificada por actividad.

\begin{tabular}{lrr}
\hline & Frecuencia & Porcentaje \\
\hline Industria & 13 & $19 \%$ \\
Energía & 12 & $18 \%$ \\
Minería & 11 & $16 \%$ \\
Financiero & 10 & $15 \%$ \\
Servicios & 10 & $15 \%$ \\
Comercial & 6 & $9 \%$ \\
Construcción & 5 & $7 \%$ \\
\hline Total & 67 & $100 \%$ \\
\hline
\end{tabular}

Fuente: Elaboración de acuerdo a base de datos 


\section{Variables}

La variable dependiente de nivel de información ambiental, comprende el cumplimiento de información de los 34 indicadores de medio ambiente de la Guía G4 de la GRI, clasificados en las categorías de gestión de materiales, agua, biodiversidad, emisiones, residuos, productos-servicios, cumplimiento regulatorio, transporte, evaluación ambiental de proveedores y mecanismos de reclamación en materia ambiental. Cada indicador se midió con el valor 0 si no cumple con la información y con valor 1 si cumple, para el análisis descriptivo. Para los reportes no GRI, se verificó la información equivalente divulgada al aspecto correspondiente a los indicadores GRI divulgados. También se obtuvieron promedios de incidencia para determinar índices de cumplimiento de las categorías de desempeño ambiental para el análisis multivariante (Rabasedas, 2018). La variable dependiente corresponde a los aspectos de prácticas de EC revelados por las empresas en sus reportes de sostenibilidad (Stewart \& Niero, 2018), se revisaron, mediante un análisis de contenido los aspectos determinados de acuerdo a la literatura revisada (Ver Tabla 1). Para evaluar la revelación de aspectos de EC se determinó una variable categórica de valor 0 cuando no menciona el aspecto, 1 si lo menciona o revela; 2 si muestra información relevante y datos cuantitativos sobre el aspecto.

Respecto a las variables independientes, el tamaño de la empresa se midió mediante el el número de trabajadores revelados en el reporte (Alonso-Almeida et al., 2015). Para la clasificación por industria se consideraron los de la Bolsa de Valores de Lima: energía, minería, construcción, industria, servicios, financiero y comercial para el análisis descriptivo, y una variable categórica la clasificación en dos grupos, uno de valor 1 para empresas con impacto de sostenibilidad importante (energía, minería, construcción e industria) y con valor 0 para las del grupo que no tenían dicho impacto (servicios, financiero y comercial) (Jackson et al., 2019).

\section{RESULTADOS}

\section{Análisis Descriptivo}

Cómo se observa en la Figura 1 el nivel de cumplimiento de divulgación de aspectos de desempeño ambiental no alcanzó el máximo nivel de información en la mayoría de sus indicadores. Las empresas estudiadas revelaron más aspectos de cumplimientos regulatorios ambientales e impacto ambiental de la actividad de transporte y la gestión de agua y residuos. La gestión con la biodiversidad y responsabilidad en producto y servicios ofrecidos fueron los indicadores con menor cumplimiento.

Respeto al sector empresarial, la Figura 2 muestra el nivel de cumplimiento de aspectos ambientales por categorías. Notamos que las empresas industriales, construcción, energía y minería fueron las que presentan un mayor índice de cumplimiento, para las prácticas relacionadas con su actividad productiva e impacto ambiental. 


\section{Figura 1}

Cumplimiento de categorías de desempeño ambiental del GRI .

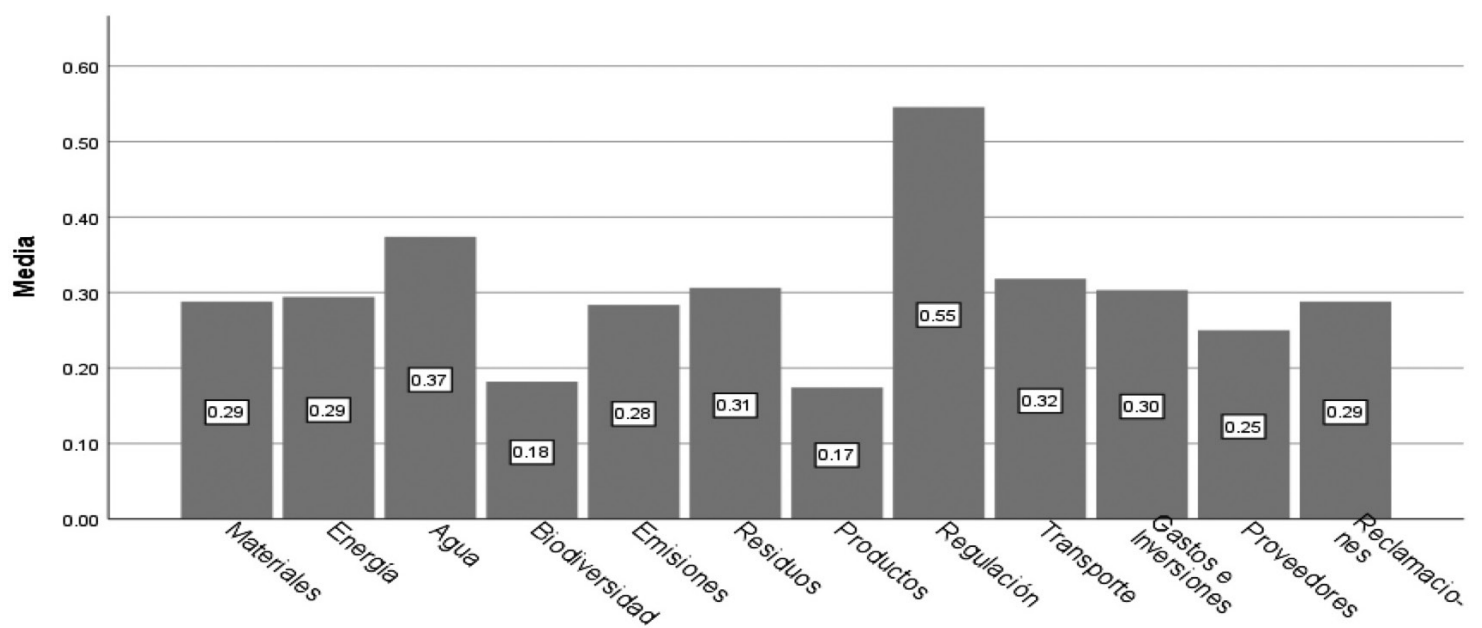

Nota. Preparado en base a datos obtenidos de las memorias analizadas

\section{Figura 2}

Índice de Cumplimento de información ambiental por actividad.

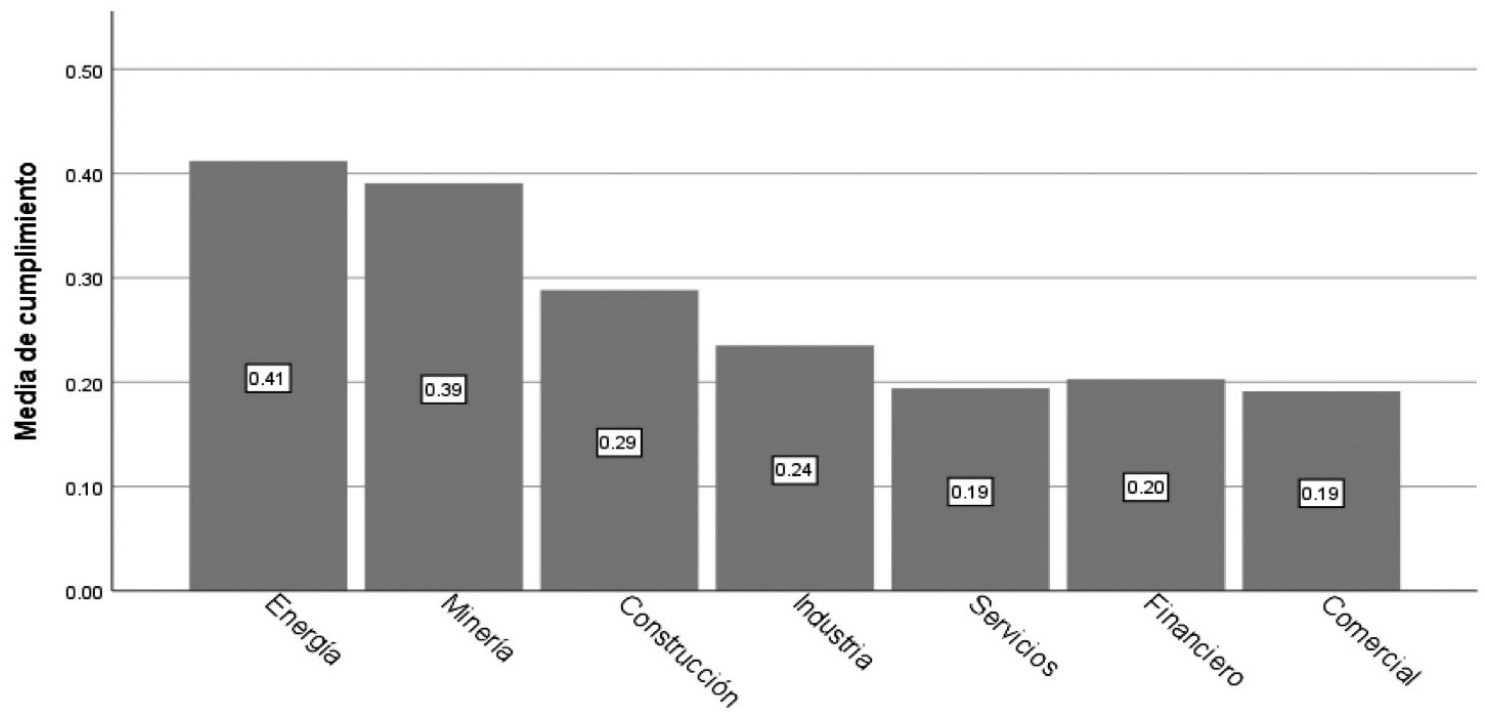

Nota. Preparado en base a datos obtenidos de las memorias analizadas 
La Figura 3 señala el nivel de cumplimento de prácticas de EC, pese a que el nivel de cumplimiento no es alto, destaca la información para las prácticas relacionadas con la reducción de consumos de insumos y recursos naturales, reducción de emisiones y reutilización de residuos generados que informaron las empresas. Por otro lado, aspectos como el cambio de consumo de recursos no renovables a renovables, uso de recursos reciclados y eco diseño, presentan un menor nivel de revelación.

\section{Figura 3}

Media de cumplimiento de Prácticas de EC.

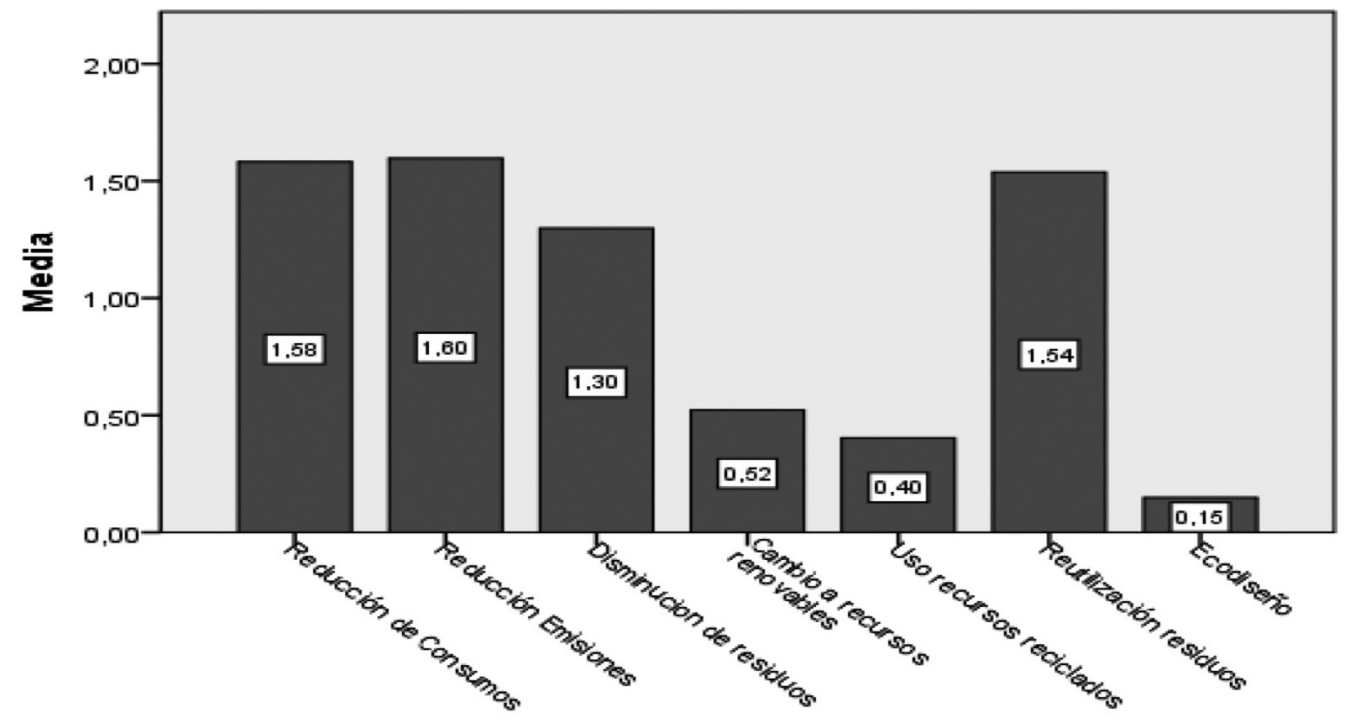

Nota. Preparado en base a datos obtenidos de las memorias analizadas

La Figura 4 no presenta diferencia significativa en el nivel de información del índice de prácticas de EC de acuerdo a la actividad empresarial, lo que difiere con el nivel de cumplimiento de desempeño ambiental que presentó un mayor nivel de divulgación para sectores con mayor impacto ambiental. 


\section{Figura 4}

Media de cumplimiento de Prácticas de EC.

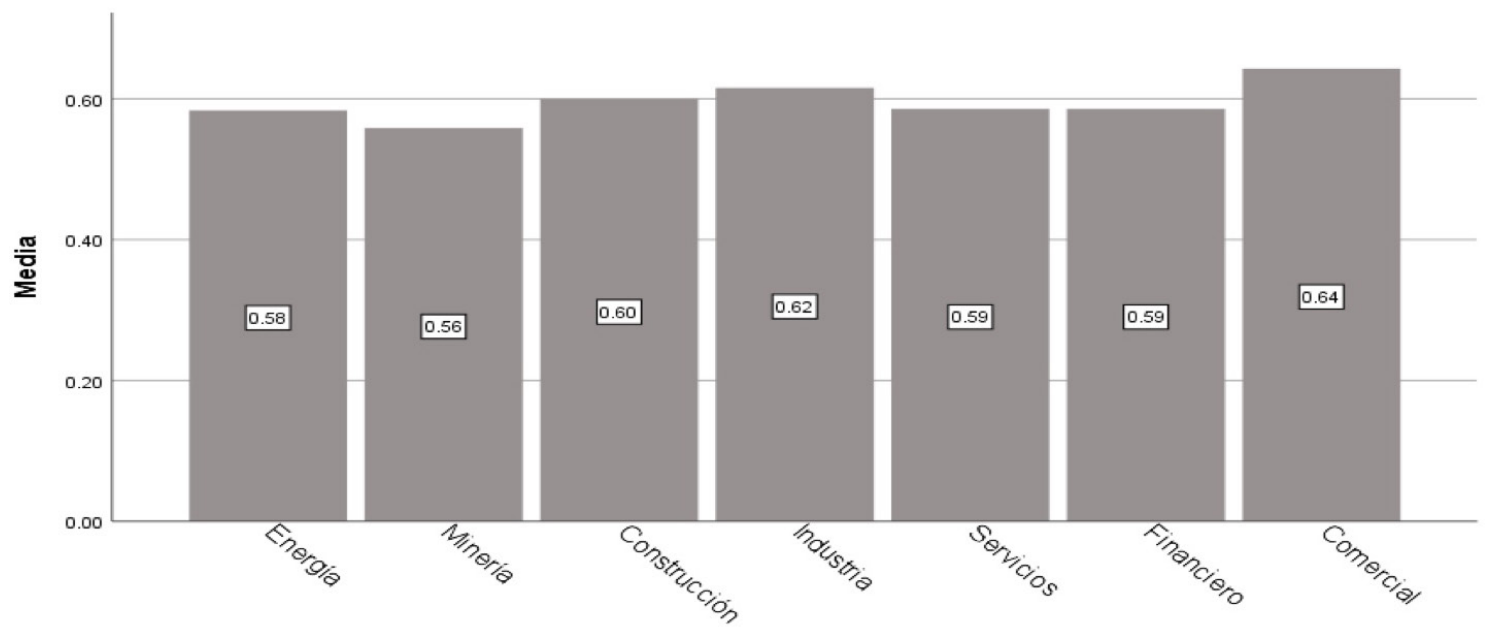

Nota. Preparado en base a datos obtenidos de las memorias analizadas

\section{Análisis relacional}

En primer lugar, se analizó si los sectores empresariales tienen incidencia significativa en los niveles de cumplimiento de desempeño ambiental de acuerdo a los aspectos del GRI y a los aspectos de prácticas de EC mediante un análisis de incidencia de la Tabla 3.

Los resultados señalan que solo el sector empresarial incide significativamente $(p<0,10)$ en el nivel de cumplimiento de desempeño ambiental de la GRI, por lo que no rechazamos la H1 para esta variable. Como observamos en el análisis descriptivo (ver figuras 1 y 2) son las empresas de sectores con importante actividad ambiental de uso de materiales, emisiones, residuos y agua, las que revelan un cumplimiento regulatorio como el caso de actividades de construcción, industriales, de energía (generación eléctrica, petróleo y gas) y minería.

En el caso de la incidencia en la información de aspectos de EC, el nivel de cumplimiento no varía significativamente de acuerdo al sector empresarial, por lo que rechazamos la H2. Las empresas no se han especializado en temas de EC.

\section{Tabla 3}

Prueba de Kruskal Wallis de incidencia del sector en los indices de cumplimiento.

\begin{tabular}{lcc}
\hline & Índice de Indicadores Ambientales & Índice de Prácticas de EC \\
\hline H de Kruskal-Wallis & 10.984 & 1.510 \\
gl & 6 & 6 \\
Sig. asintótica & $.089^{*}$ & .959 \\
\hline${ }^{*} \mathrm{p}<0,10$ & &
\end{tabular}

Nota. Prueba elaborada de acuerdo a base de datos 
Finalmente, para evaluar la correlación entre las variables independientes de tamaño y sector con las variables dependientes de nivel de cumplimiento de aspectos ambientales de la GRI y nivel de información de prácticas de EC, se realizó una correlación de Spearman, cuyos resultados se observan en la Tabla 4.

El tamaño de la empresa presenta solo una relación negativa y significativa $(p<0,01$ y $p<0,05)$ con algunos aspectos de cumplimiento regulatorio, atención de reclamos e inversiones ambientales. Rechazamos la H3 para esta variable pues se esperaba que las empresas más grandes y con mayores trabajadores muestren un mayor cumplimiento de indicadores ambientales en todas sus categorías, y no es así, en algunos casos las empresas de gran tamaño no muestran un alto grado de divulgación.

En la matriz de correlaciones se observa, que el sector empresarial se relaciona de forma significativa y positiva $(\mathrm{p}<0,01$ y $\mathrm{p}<0,05)$ con algunos aspectos de desempeño ambiental, por lo que no rechazamos la H3 para dicha variable. Es así que las empresas del grupo de mayor impacto ambiental (agroindustria, energía, industria, minería) se relacionan con un importante nivel de cumplimiento de información en aspectos de gestión de agua, residuos e impactos con biodiversidad; cumplimiento regulatorio ambiental y atención de reclamaciones; e información sobre gastos e inversiones ambientales, propios de empresas de explotación de recursos e impacto ambiental en su producción.

La Tabla 5 muestra que ni el tamaño ni actividad empresarial se relacionan significativamente con la información sobre aspectos de EC, por lo que rechazamos la H4. Los resultados señalan que la información de actividades de EC no solo corresponde a algunas grandes empresas, sino a otras de menor tamaño. 


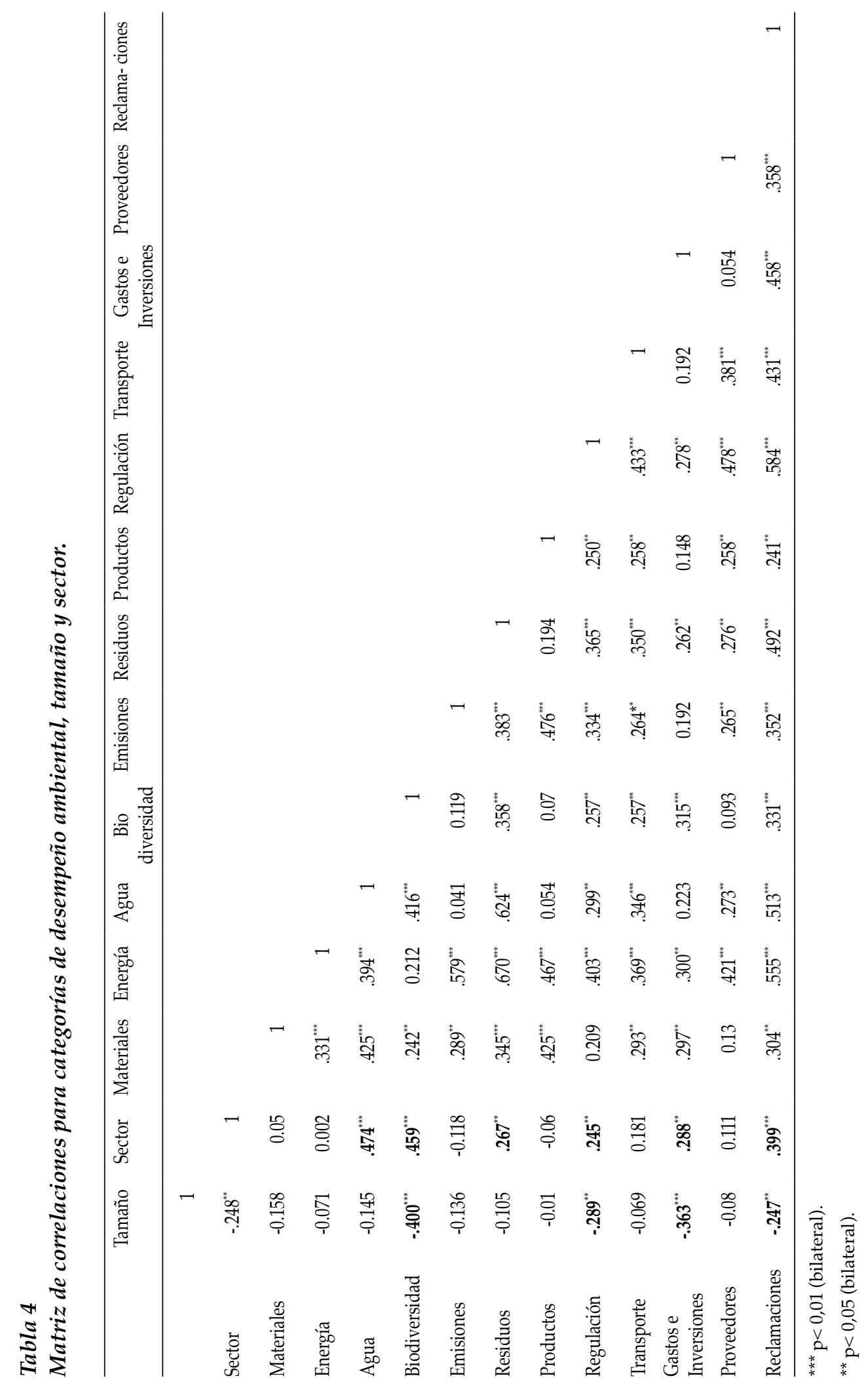




\section{Tabla 5}

Matriz de correlaciones para aspectos de EC, tamaño y sector.

\begin{tabular}{|c|c|c|c|c|c|c|c|c|c|}
\hline & Tamaño & Sector & $\begin{array}{l}\text { Reducción } \\
\text { Consumos }\end{array}$ & $\begin{array}{l}\text { Reducción } \\
\text { Emisiones }\end{array}$ & $\begin{array}{l}\text { Disminución } \\
\text { residuos }\end{array}$ & $\begin{array}{c}\text { Cambio a } \\
\text { recursos } \\
\text { renovables }\end{array}$ & $\begin{array}{l}\text { Uso } \\
\text { recursos } \\
\text { reciclados }\end{array}$ & $\begin{array}{l}\text { Reutilización } \\
\text { residuos }\end{array}$ & $\begin{array}{l}\text { Eco } \\
\text { diseño }\end{array}$ \\
\hline Tamaño & 1.000 & & & & & & & & \\
\hline SectorVibariado & $-.248^{* *}$ & 1.000 & & & & & & & \\
\hline $\begin{array}{l}\text { Reducción de } \\
\text { Consumos }\end{array}$ & 0.143 & -0.032 & 1.000 & & & & & & \\
\hline $\begin{array}{l}\text { Reducción } \\
\text { Emisiones }\end{array}$ & 0.024 & 0.021 & $.395^{*+* x}$ & 1.000 & & & & & \\
\hline $\begin{array}{l}\text { Disminucion de } \\
\text { residuos }\end{array}$ & 0.032 & 0.022 & $.334^{+3+x}$ & 0.190 & 1.000 & & & & \\
\hline $\begin{array}{l}\text { Cambio a recursos } \\
\text { renovables }\end{array}$ & -0.158 & 0.054 & 0.104 & 0.044 & -0.057 & 1.000 & & & \\
\hline $\begin{array}{l}\text { Uso recursos } \\
\text { reciclados }\end{array}$ & 0.044 & 0.077 & 0.109 & 0.129 & $.327^{\text {stot }}$ & 0.028 & 1.000 & & \\
\hline $\begin{array}{l}\text { Reutilización } \\
\text { residuos }\end{array}$ & -0.016 & -0.092 & 0.175 & 0.009 & $.266^{* *}$ & -0.028 & 0.087 & 1.000 & \\
\hline Ecodiseño & 0.025 & -0.007 & 0.126 & -0.009 & 0.191 & 0.129 & 0.137 & 0.117 & 1.000 \\
\hline
\end{tabular}

\section{DISCUSION}

Esta investigación busca aportar a la investigación sobre la naturaleza y factores de información medioambiental y de prácticas de EC, divulgados en reportes de sostenibilidad de empresas peruanas. Para el periodo estudiado los resultados señalan que dichos reportes han presentado un importante cumplimiento en determinados indicadores ambientales, como la gestión de residuos, agua, transporte, y cumplimiento regulatorio, desde un punto de vista institucional como un cumplimento de la normativa del GRI y de la regulación ambiental para sectores específicos (Hahn \& Kühnen, 2013; Kuzey \& Uyar, 2016). Otros aspectos de desempeño como la responsabilidad de productos, gestión de emisiones, energía, materiales y con proveedores no están muy desarrollados ni se muestra un enfoque de gestión con los grupos de interés (Calixto, 2013; Del Barco et al., 2018; Hernández-Pajares, 2018).

Sin embargo, empresas de energía, mineras, industria y construcción, que tienen un mayor impacto ambiental, disponibilidad de recursos y relación con las comunidades donde operan, son las que presentan el mayor cumplimiento como una forma de legitimación para cumplir con 
información exigida por entes reguladores en mercados de valores y responder a la expectativa de las comunidades y sociedad respecto al impacto ambiental de sus actividades, principalmente en actividades de energía (electricidad, petróleo y gas) y minería (Cormier y Magnan, 2003; Da Silva \& Aibar, 2011; Bednárová et al., 2019; Jenkins \& Yakovleva; 2006).

Respecto a la EC, aún no se encuentra un desarrollo en las empresas estudiadas las cuales no señalan explícitamente una estrategia y gestión de EC (Kirchherr et al., 2017; Veleva et al., 2017; Stewart y Niero, 2018). La información descrita en los informes corresponde solo a prácticas reactivas y resultan insuficientes para respaldar una gestión integral e innovadora de EC (Moneva et al, 2018; Veleva et al., 2017; Janik et al., 2020), y resultan un mero cumplimiento normativo de prácticas como reducción de emisiones y consumos y reutilización de residuos (Aranda-Usón et al., 2020; Ghisellini et al., 2016; Moneva et al, 2018; Scarpellini et al., 2019).

Esto implica un mayor apoyo de las asociaciones empresariales y el gobierno en incentivar modelos de gestión sostenibles en las empresas. Resulta necesario una mayor influencia institucional, mediante incentivos y regulaciones, por parte de entidades gubernamentales y privadas para mejorar el desempeño ambiental e implementación de modelos de negocio innovadores según la EC de las organizaciones para grandes y pequeñas empresas (Aranguren \& Maldonado, 2019; Janik et al., 2020; Ormazabal et al., 2018).

\section{REFERENCIAS BIBLIOGRÁFICAS}

Alonso-Almeida, M., Marimon, F., \& Llach, J. (2015). Difusión de las memorias de sostenibilidad en Latinoamérica: análisis territorial y sectorial. Estudios Gerenciales, 31, 139-149.

Alvarez-Risco, A., Rosen, M. A., \& Del-Aguila-Arcentales, S. (2020). A New Regulation for Supporting a Circular Economy in the Plastic Industry: The Case of Peru. Journal of Landscape Ecology, 13(1), 1-3.

Alvarez-Risco, A., Estrada-Merino, A., Rosen, M. A., Vargas-Herrera, A., \& Del-Aguila-Arcentales, S. (2021). Factors for implementation of circular economy in firms in covid-19 pandemic times: The case of Peru. Environments, 8(9), 95.

Aranda-Usón, A., Portillo-Tarragona, P., Scarpellini, S., \& Llena-Macarulla, F. (2020). The progressive adoption of a circular economy by businesses for cleaner production: An approach from a regional study in Spain. Journal of Cleaner Production, 247, 119648.

Aranguren, N., \& Maldonado, S. (2019). Governance and Type of Industry as Determinants of Corporate Social Responsibility Disclosures in Latin America. Latin American Business Review, 21 (1), 1-35

Baalouch, F., Damak, S., \& Hussainet, K. (2019). A study of the determinants of environmental disclosure quality: evidence from French listed companies. Journal of Management and Governance, 23(4), 939-971.

Barnabè, F., \& Nazir, S. (2020). Investigating the interplays between integrated reporting practices and circular economy disclosure. International Journal of Productivity and Performance Management, 70(8), 2001-2031.

Bednárová, M., Klimko, R., \& Rievajová, E. (2019). From Environmental Reporting to Environmental Performance. Sustainability, 11(9), 1-12. 
Bhattacharyya, A. (2014) Factors Associated with the Social and Environmental Reporting of Australian Companies, Australasian Accounting, Business and Finance Journal, 8(1), 25-50.

Bocken, N., De Pauw, I., Bakker, C., \& Van der Grinten, B. (2016). Product design and business model strategies for a circular economy. Journal of Industrial and Production Engineering, 33(5), 308-320.

Calixto, L. (2013). A divulgação de relatórios de sustentabilidade na América Latina: um estudo comparativo. Revista de Administração, 48(4), 828-842.

Cerdá, E., y Khalilova, A. (2016). Economía circular. Economía industrial, 401, 11-20.

Cormier, D., Magnan, M., \& Van Velthoven, B. (2005). Environmental disclosure quality in large German companies: economic incentives, public pressures or institutional conditions?. European accounting review, 14(1), 3-39.

Cormier, D., \& Magnan, M. (2003). Environmental reporting management: a continental European perspective. Journal of Accounting and public Policy, 22(1), 43-62.

D’Amico, E., Coluccia, D., Fontana, S., \& Solimene, S. (2016). Factors Influencing Corporate Environmental Disclosure. Business Strategy and the Environment. 25(3), 178-192.

Da Silva, S.M., \& Aibar, B. (2011). Factores Determinantes del grado de información medioambiental divulgada en las grandes empresas que operan en Portugal: Un análisis univariante. Revista de Administração e Contabilidade da Unisinos, 8(1), 3-19.

Del Barco, J. P., Del Barco, M. A., Del Barco, M. S., \& Federici, I. (2018). Divulgación de aspectos ambientales en los informes de sostenibilidad frente al nuevo escenario internacional. $C A$ PIC REVIEW, 16, 1-19.

Deegan, C. (2002). The legitimising effect of social and enviromental disclousure - a theoretical foundation. Accounting, Auditing \& Accountability Journal, 15(3), 282-311.

De Villiers, C., \& Alexander, D. (2014). The institutionalisation of corporate social responsibility reporting. The British Accounting Review, 46(2), 198-212.

Dyduch, J., \& Krasodomska, J. (2017). Determinants of Corporate Social Responsibility Disclosure: An Empirical Study of Polish Listed Companies. Sustainability 9(11), 1-24.

Elia, V., Gnoni, M.G., \& Tornese, F. (2017). Measuring circular economy strategies through index methods: A critical analysis. Journal of Cleaner Production, 142, 2741-2751

Geng, Y., Fu, J., Sarkis, J., \& Xue, B. (2012). Towards a national circular economy indicator system in China: an evaluation and critical analysis. Journal of Cleaner Production, 23, 216-224.

Geissdoerfer, M., Savaget, P., Bocken, N. M., \& Hultink, E. J. (2017). The Circular Economy-A new sustainability paradigm?. Journal of Cleaner Production, 143, 757-768.

Ghisellini, P., Cialani, C., Ulgiati, S. (2016). A review on circular economy: the expected transition to a balanced interplay of environmental and economic systems. Journal of Cleaner Production, 114, 11-32.

Hahn, R., \& Kühnen, M. (2013). Determinants of sustainability reporting: a review of results, trends, theory, and opportunities in an expanding field of research. Journal of cleaner production, 59, 5-21.

Hernández-Pajares, J. Venegas, C., \& Alvarado, L. (2017). Factores medioambientales de las empresas mineras peruanas. CAPIC REVIEW, 14 (1), 29-37.

Hernández-Pajares, J. (2018). Influencia de la naturaleza internacional de empresas peruanas en su información de sostenibilidad. Revista de Comunicación 17(1). 74-92

Husillos, J. (2007). Una aproximación desde la teoría de la legitimidad a la información medioambiental revelada por las empresas españolas cotizadas. Spanish Journal of Finance and Accounting, 36(133), 97-121. 
Jackson, G., Bartosch, J., Avetisyan, E., Kinderman, D., \& Knudsen, J. (2019). Mandatory non-financial disclosure and its influence on CSR: An international comparison. Journal of Business Ethics, 1-20.

Janik, A., Ryszko, A., \& Szafraniec, M. (2020). Greenhouse gases and circular economy issues in sustainability reports from the energy sector in the European Union. Energies, 13(22), 5993.

Jenkins, H., \& Yakovleva, N. (2006). Corporate social responsibility in the mining industry: Exploring trends in social and environmental disclosure. Journal of cleaner production, 14(3-4), 271-284.

Kirchherr, J., Reike, D., \& Marko, H. (2017). Conceptualizing the circular economy: An analysis of 114 definitions. Resources, Conservation \& Recycling, 127, 221-232.

Kolk, A., Walhain S., \& Van de Wateringen. (2001). Environmental reporting by the Fortune Global 250: eExploring the influence of nationality and sector. Business Strategy and the Environment, 10 (1), 15-28.

Kouloukoui, D., Oliveira, Â. M., Da Silva, S.M., De Oliveira, M. M., De Jong, P., Kiperstok, A., y Andrade, E. (2018). Factors influencing the level of environmental disclosures in sustainability reports: Case of climate risk disclosure by Brazilian companies. Corporate Social Responsibility and Environmental Management, 26(4), 1-14.

Kuo, L., \& Chang, B. G. (2021). The affecting factors of circular economy information and its impact on corporate economic sustainability-Evidence from China. Sustainable production and consumption, 27, 986-997.

Kuzey, C. \& Uyar, A. (2017). Determinants of sustainability reporting and its impact on firm value: evidence from the emerging market of Turkey. Journal of Cleaner Production, 143, 27-39.

Lacy, P., \& Rutqvist, J. (2015). Waste to Wealth: The Circular Economy Advantage. Palgrave Macmillan, New York.

Landrum, N. E., \& Ohsowski B. (2018). Identifying worldviews on corporate sustainability: a content analysis of corporate sustainability reports. Business Strategy and the Environment, 27(1), 128-151.

Landrum, Nancy E. (2017). Stages of corporate sustainability: integrating the strong sustainability worldview. Organization \& Environment, 1-27.

Laso, J., García-Herrero, I., Margallo, M., Vázquez-Rowe, I., Fullana, P., Bala, A., Gazullad, C. Irabiena, A., Aldacoa R. (2018). Finding an economic and environmental balance in value chains based on circular economy thinking: An eco-efficiency methodology applied to the fish canning industry. Resources, Conservation and Recycling, 133, 428-437.

Li, H., Bao, W., Xiu, C., Zhang, Y., \& Xu, H. (2010). Energy conservation and circular economy in China's process industries. Energy, 35(11), 4273-4281.

Luttropp, C., \& Lagerstedt, J. (2006). EcoDesign and The Ten Golden Rules: generic advice for merging environmental aspects into product development. Journal of Cleaner Production, 14, 1396-1408.

Linder, M., \& Williander, M. (2015). Circular Business Model Innovation: Inherent Uncertainties. Business Strategy and the Environment, 26(2), 182-196.

Marimon, F. Alonso-Almeida, M., Rodríguez, M., \& Alejandro, K. (2012). The worldwide diffusion of the global reporting initiative: what is the point?. Journal of Cleaner Production, 33, 132-144.

Moneva, J.M., Portillo-Tarragona, P., Llena-Macarulla, F., \& Scarpellini, S. (2018). Perspectivas e impacto de la Economía Circular en Aragón desde la óptica empresarial. Economía aragonesa, 66, 111-126. 
Moneva J.M., Jara-Sarrúa L., Hernández-Pajares J. \& Del Barco J. (2019). The disclosure of social issues in Latin American sustainability reports: an exploration of their disclosure in Argentina, Chile, and Peru. En Corporate Social Responsibility Disclosure and Assurance: A Growing Market. Cambridge Scholars Publishing, 135-174.

Murray, A., Skene, K., \& Haynes, K. (2017). The circular economy: An interdisciplinary exploration of the concept and application in a global context. Journal of Business Ethics, 140(3), 369-380

Neu, D., Warsame, H. \& Pedwell, K. (1998) Managing public impressions: environmental disclosures in annual reports, Accounting, Organizations and Society, 23(3), 265-282.

Orazalin, N., \& Mahmood., M. (2019). Determinants of GRI-based sustainability reporting: evidence from an emerging economy. Journal of Accounting in Emerging Economies. 10 (1), 140164.

O'Donovan, G. (2002). Environmental disclosures in the annual report. Extending the applicability and predictive power of legitimacy theory, Accounting, Auditing and Accountability Journal, 15 (3), 344-37.

Ormazabal, M., Prieto-Sandoval, V., Puga-Leal, R., \& Jaca, C. (2018). Circular Economy in Spanish SMEs: Challenges and opportunities. Journal of Cleaner Production, 185, 157-167.

Paño-Yáñez, P. (2021). Viabilidad de la economía circular en países no industrializados y su ajuste a una propuesta de economías transformadoras. Un acercamiento al escenario latinoamericano, CIRIEC-España, Revista de Economía Pública, Social y Cooperativa, 101, 289-323.

Potting, J., Hekkert, M., Worrell, E., \& Hanemaaijer, A. (2017). Circular economy: measuring innovation in the product chain. PBL Netherlands Environmental Assessment Agency.

Prieto-Sandoval, V., Jaca, C., \& Ormazabal, M. (2018). Towards a consensus on the circular economy. Journal of Cleaner Production, 179, 605-615.

PRODUCE (2020). Decreto Supremo No 003-2020-PRODUCE, Ministerio de Producción. Hoja de Ruta hacia una Economía Circular en el Sector Industria. https: / /www.gob.pe/institucion / produce/ normas-legales / 444490-003-2020-produce

Rabasedas, M., Del Barco, M., \& Jara-Sarrúa, L. (2016). Análisis intersectorial de las memorias de sostenibilidad publicadas por empresas cotizadas en Argentina. SaberEs, 8(2), 133-160.

Rabasedas, M. (2018). Divulgación de información medioambiental: análisis exploratorio para las entidades cotizadas en Argentina. CAPIC REVIEW, 16, 1-16.

Scarpellini, S., Portillo-Tarragona, P., Aranda-Usón, A., \& Llena-Macarulla, F. (2019). Definition and measurement of the circular economy's regional impact. Journal of Environmental Planning and Management, 62(13), 2211-2237.

Sehnem, S., Santos, A. A. P., \& Silva, C. G. (2019). Is the sustainability a driver of the circular economy? Social Responsibility Journal, 1, p. 1747-1117.

Stewart, R., \& Niero, M. (2018). Circular economy in corporate sustainability strategies: A review of corporate sustainability reports in the fast-moving consumer goods sector. Business Strategy and the Environment, 27(7), 1005-1022.

Sustainability Disclosure Database. (GRI, 2019). https:/ / database.globalreporting.org/

Veleva, V., Bodkin, G., \& Todorova, S. (2017). The need for better measurement and employee engagement to advance a circular economy: Lessons from Biogen's "zero waste” journey. Journal of cleaner production, 154, 517-529.

Wang, P., Che, F., Fan, S. \& Gu, C. (2014). Ownership governance, institutional pressures and circular economy accounting information disclosure an institutional theory and corporate governance theory perspective. Chinese Management Studies, 8 (3), 487-501. 
Yuan, Z., Bi, J., \& Moriguichi, Y. (2006). The circular economy: A new development strategy in China. Journal of Industrial Ecology, 10(1-2), 4-8.

\section{(c)}

Esta obra está bajo una licencia de Creative Commons Atribución-NoComercial-CompartirIgual 4.0 Internacional 